\title{
Magnetohydrodynamics (MHD) stagnation point flow past a shrinking/ stretching surface with double stratification effect in a porous medium
}

\begin{abstract}
The present work emphasizes the MHD mixed convective stagnation point flow over a shrinking/stretching surface saturated in a porous medium. The double stratification with heat source effects are also considered while the magnetic field is imposed normal to the sheet. The governing model (partial differential equations) is converted into a system of ordinary (similarity) differential equations using similarity transformations. The boundary value problem solver (bvp4c) in the MATLAB software is utilized for the numerical computations. Numerical results are graphically illustrated in the form of velocity, temperature and concentration profiles for several values of buoyancy, magnetic, thermal and solutal stratification parameters. The graphs of skin friction coefficient, local Nusselt and Sherwood numbers portray that the dual solutions are achievable within a certain range of the buoyancy and velocity ratio parameters. Both assisting and opposing flow cases can generate two solutions, whereas the forced convective flow only produces a unique solution. The execution of stability analysis affirms the reliability of the first solution. Both heat and mass transfer rates intensify with the increment of the velocity ratio parameter for all type of convective flows. The fluid temperature and concentration decrease with the increment of the thermal and solutal stratification parameters, respectively, whereas the magnetic and buoyancy parameters reduce both temperature and concentration profiles.
\end{abstract}

Keyword: Stagnation point flow; Stretching/shrinking sheet; Magnetohydrodynamics; Double stratification; Porous medium; Dual solutions 